\title{
Fission barriers and half-lives of actinides in the quasi-molecular shape valley
}

\author{
G. Royer, M. Jaffré, D. Moreau \\ Laboratoire Subatech, UMR: IN2P3/CNRS-Université-Ecole des Mines, Nantes 44, France
}

\begin{abstract}
The energy of actinide nuclei in the fusionlike deformation valley has been determined from a liquid drop model taking into account the proximity energy, the mass and charge asymmetries and the shell and pairing energies. Double-humped potential barriers appear. The saddle-point corresponds to the second maximum and to the transition from compact one-body shapes with a deep neck to two touching ellipsoids. The scission point, where the effects of the nuclear attractive forces between the fragments vanish, lies at the end of an energy plateau below the saddle-point and corresponds to two well separated fragments. The kinetic and excitation energies of the fragments come from the energy on this plateau. The shell and pairing effects play a main role to decide the most probable decay path. The heights of the potential barriers roughly agree with the experimental data and the calculated half-lives follow the trend of the experimental values. A shallow third minimum and a third peak appear in specific asymmetric exit channels where one fragment is close to a double magic quasi-spherical nucleus, while the other one evolves from oblate to prolate shapes.
\end{abstract}

PACS numbers: 25.85.Ca,24.75.+i,21.60.Ev,21.10.Tg

\section{INTRODUCTION}

The observed properties of the ground state and isomeric state spectra of actinides suggest the existence of doublehumped potential barriers. The heights of the inner and asymmetric outer fission barriers are almost constant (5-6 $\mathrm{MeV}$ ) from Th to Am isotopes [1, 2]. This fact is a severe test for the theoretical models. Furthermore, the analysis of the fission probability and of the angular distribution of the fragments indicate the existence of hyperdeformed states in a deep third well in several Th and U isotopes [3-5] confirming the pioneering work of Blons et al [6] in ${ }^{231,233} \mathrm{Th}$. The observed strongly enhanced low energy $\alpha$ decay of several heavy actinide nuclei is also understood assuming the decay of a third hyperdeformed minimum and the possibility that the third minimum is the true ground state of very heavy and perhaps superheavy nuclei has been also advocated [7]. Beyond the actinides, and very surprisingly, it is also necessary to advance fission barrier heights of $5-10 \mathrm{MeV}$ to explain the successfull formation in heavy ion reactions of superheavy elements of charge 112-118, pointing out the determining influence of the shell effects [8,9].

The fission shapes were firstly investigated long time ago by minimizing the sum of the Coulomb and surface energies using mainly a development of the radius in Legendre polynomials. This leads naturally to fission paths through very elongated shapes with shallow neck or no neck. By adding to the macroscopic Liquid Drop Model energy of these elongated one-body shapes an oscillatory microscopic contribution, the Strutinsky's method [10] generated doublehumped barriers allowing to explain the fission isomer characteristics. On the other side, Myers and Swiatecki [11] proposed analytical formulae to calculate rapidly the shell and pairing energies for deformed shapes. Later on, the asymmetric two-center shell model [12], the Hartree-Fock-Bogoliubov [13] and the relativistic mean field theories [14] have also succeeded in obtaining different minima in the potential landscape where strongly deformed fission isomers can survive.

Within a Generalized Liquid Drop Model (GLDM) taking into account both the proximity energy between close opposite surfaces and the mass and charge asymmetries, most of symmetric and asymmetric fission [15-17], $\alpha$ and light nucleus emission [18, 19], and fusion data [20] have been also reproduced in the fusionlike shape valley as well as the rotating super and highly deformed state characteristics [21, 22]. It has been proved that there is a degeneracy between the energy of elongated shapes with a shallow neck and the energy of quasi-molecular shapes, as well as between the values of the moment of inertia and quadrupole moment [23] in the two deformation paths. Consequently, the experimental data do not allow us to choose between them.

The purpose of this work is to focus on the actinide region remaining in the peculiar quasi-molecular shape valley, taking into account the ellipsoidal deformations of the two different fission fragments, in order to investigate all possible mass and charge asymmetries in the framework of the GLDM, accounting for shell and pairing energies. The study is limited to quasi-molecular shapes since these shapes are rarely investigated and are inaccessible in using the usual development of the nuclear radius. 


\section{POTENTIAL ENERGY OF A DEFORMED NUCLEUS}

The energy of a deformed nucleus is the sum of the GLDM energy and the microscopic shell and pairing energies. The GLDM energy is given by [20]

$$
E=E_{V}+E_{S}+E_{C}+E_{\text {prox }}
$$

where the different terms are, respectively, the volume, surface, Coulomb and nuclear proximity energies.

All along the fission path the proximity energy term $E_{\text {prox }}$ takes into account the nuclear attractive forces between nucleons in the neck, in the case of a deformed one-body shape, or across the gap, in the case of two separated fragments. In the quasi-molecular shape valley where the necks are narrow and well developed this correction to the surface energy plays a main role on a large part of the fission path and specially around the touching point. For example, at the contact point between two spherical $\mathrm{Kr}$ and Ba nuclei the proximity energy reaches $-43 \mathrm{MeV}$. The absence of this term in fusion studies leads to an unrealistic Coulomb peak. When the proximity energy is taken into account, the fusion barrier is smooth and the maximum corresponds to two separated nuclei maintained in unstable equilibrium by the balance between the repulsive Coulomb forces and the attractive nuclear proximity forces. In fission studies this term is very important when creviced and compact shapes are considered and is almost negligible for elongated shapes with a shallow neck. The proximity energy is defined as :

$$
E_{\text {prox }}(r)=2 \gamma \int \Phi[D(r, h) / b] 2 \pi h d h
$$

where $r$ is the distance between the mass centres. $\Phi$ is the proximity function of Feldmeier [24]. $h$ is the transverse distance varying from zero, for separated fragments or the neck radius for one-body shapes, to the height of the neck border. $b$ the surface width fixed at the standard value of $0.99 \mathrm{fm}$. $D$ is the distance between the opposite surfaces on a line parallel to the fission axis (see also $[20,24]$ ). Finally the surface parameter $\gamma$ is given by a geometric mean between the surface parameters of the two fragments :

$$
\gamma=0.9517\left(1-k_{s} I_{1}^{2}\right)^{1 / 2}\left(1-k_{s} I_{2}^{2}\right)^{1 / 2} M e V \cdot f m^{-2} .
$$

The selected one-body shape sequence simulating the fusionlike fission valley is shown in the upper part of Fig. 1. Two elliptic lemniscatoids are connected allowing the development of a deep neck while keeping almost spherical ends [20]. The proximity energy is maximized in this deformation path.

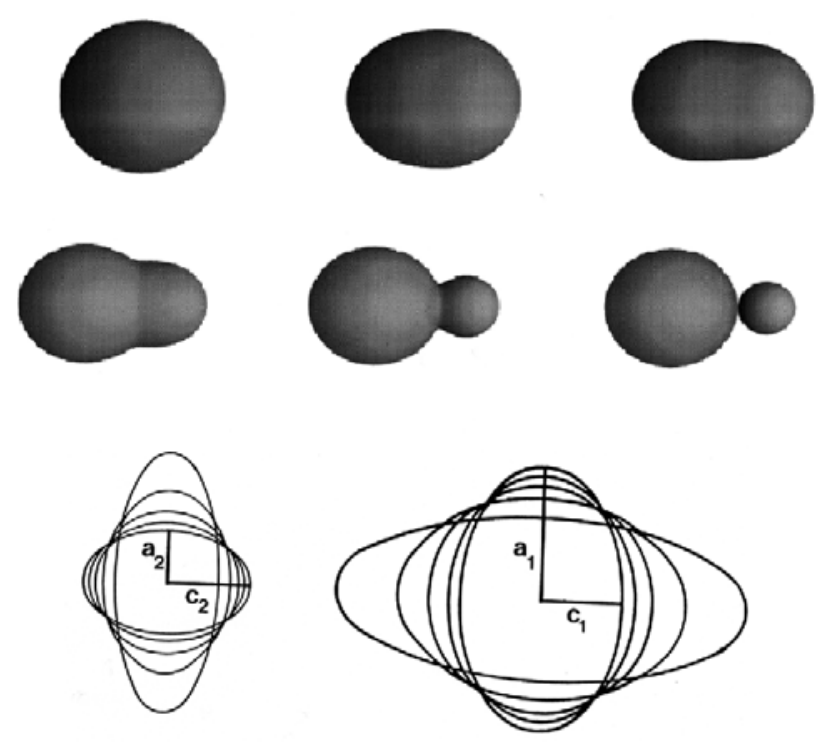

FIG. 1: Shape sequences describing the one-body shape evolution (for a given final asymmetry) and the two coaxial ellipsoid configurations. The fission axis is the common axis of revolution.

For a given final asymmetry, there is a one-to-one correspondence between the distance $r$ between the centres of the future fragments and the shape of the deformed nucleus [20]. 
For one-body shapes, the first three contributions are expressed as

$$
\begin{gathered}
E_{V}=-15.494\left(1-1.8 I^{2}\right) A M e V, \\
E_{S}=17.9439\left(1-2.6 I^{2}\right) A^{2 / 3} \frac{S}{4 \pi R_{0}^{2}} M e V,
\end{gathered}
$$

$I$ is the relative neutron excess and $\mathrm{S}$ is the surface of the deformed nucleus. The values of the surface energy coefficient and asymmetry surface energy coefficient are very close to those proposed by the Lund group and have never been changed since the first study [15].

$$
E_{C}=0.6 e^{2}\left(Z^{2} / R_{0}\right) B_{C}
$$

The Coulomb shape dependent function $B_{C}$ is defined as

$$
B_{C}=\frac{15}{16 \pi^{2} R_{0}^{5}} \int d \tau \int \frac{d \tau^{\prime}}{\left|r-r^{\prime}\right|}
$$

It has been determined within the method proposed by Cohen and Swiatecki [25] using the axial symmetry of the system and complete elliptic integrals [26]

$$
B_{C}=0.5 \int\left(V(\theta) / V_{0}\right)\left(R(\theta) / R_{0}\right)^{3} \sin \theta d \theta
$$

where $V(\theta)$ is the electrostatic potential at the surface and $V_{0}$ the surface potential of the sphere.

The radius $R_{0}$ of the compound nucleus is given by:

$$
R_{0}=\left(1.28 A^{1 / 3}-0.76+0.8 A^{-1 / 3}\right) f m .
$$

This formula proposed in Ref. [27] was derived from the Droplet Model and from the proximity energy values and simulates rather a central radius for which $R_{0} / A^{1 / 3}$ increases slightly with the mass. It has been shown that this GLDM can be used to reproduce accurately the fusion [20, 22], fission [15, 17] and cluster [19] and alpha [18] radioactivity data. Furthermore, the values of the parameters can be used to calculate the nuclear masses [28].

The radii of the two fragments are calculated assuming volume conservation. For two-body shapes, the coaxial ellipsoidal deformations of the two fragments have been considered [29] (see the lower part of Fig. 1). For a given distance $\mathrm{r}$ between the mass centers of the two fragments the system configuration depends on two parameters : the ratios $s_{i}(i=1,2)$ between the transverse semi-axis $a_{i}$ and the radial semi-axis $c_{i}$ of the fragments.

$$
a_{i}=R_{i} s_{i}^{1 / 3}, \quad c_{i}=R_{i} s_{i}^{-2 / 3}
$$

The prolate deformation is characterized by $s \leq 1$ and the related eccentricity is $e^{2}=1-s^{2}$, while in the oblate case $s \geq 1$ and $e^{2}=1-s^{-2}$.

The volume and surface energies are the sum of the contributions of each fragment.

In the prolate case, the relative surface energy is given by

$$
B_{S i}=\frac{\left(1-e_{i}^{2}\right)^{1 / 3}}{2}\left[1+\frac{\sin ^{-1}\left(e_{i}\right)}{e_{i}\left(1-e_{i}^{2}\right)^{1 / 2}}\right]
$$

and in the oblate case

$$
B_{S i}=\frac{\left(1+\epsilon_{i}^{2}\right)^{1 / 3}}{2}\left[1+\frac{\ln \left(\epsilon_{i}+\left(1+\epsilon_{i}^{2}\right)^{1 / 2}\right)}{\epsilon_{i}\left(1+\epsilon_{i}^{2}\right)^{1 / 2}}\right] \quad \epsilon_{i}^{2}=s_{i}^{2}-1 .
$$

The Coulomb self-energy of the spheroid $i$ reads

$$
E_{C, \text { self }}=\frac{3 e^{2} Z_{i}^{2} B_{c i}}{5 R_{i}}
$$


The relative self-energy is, in the prolate case

$$
B_{C i}=\frac{\left(1-e_{i}^{2}\right)^{1 / 3}}{2 e_{i}} \ln \frac{1+e_{i}}{1-e_{i}}
$$

and, in the oblate case

$$
B_{C i}=\frac{\left(1+\epsilon_{i}^{2}\right)^{1 / 3}}{\epsilon_{i}} \tan ^{-1} \epsilon_{i}
$$

The Coulomb interaction energy between the two fragments is calculated as

$$
E_{C, \text { int }}=\frac{e^{2} Z_{1} Z_{2}}{r}\left[s\left(\lambda_{1}\right)+s\left(\lambda_{2}\right)-1+S\left(\lambda_{1}, \lambda_{2}\right)\right] \quad \lambda_{i}^{2}=\frac{c_{i}^{2}-a_{i}^{2}}{r^{2}} .
$$

In the prolate case, $s\left(\lambda_{i}\right)$ is expressed as

$$
s\left(\lambda_{i}\right)=\frac{3}{4}\left(\frac{1}{\lambda_{i}}-\frac{1}{\lambda_{i}^{3}}\right) \ln \left(\frac{1+\lambda_{i}}{1-\lambda_{i}}\right)+\frac{3}{2 \lambda_{i}^{2}},
$$

while, for the oblate shapes,

$$
s\left(\lambda_{i}\right)=\frac{3}{2}\left(\frac{1}{\omega_{i}}+\frac{1}{\omega_{i}^{3}}\right) \tan ^{-1} \omega_{i}-\frac{3}{2 \omega_{i}^{2}} \quad \omega_{i}^{2}=-\lambda_{i}^{2} .
$$

$S\left(\lambda_{1}, \lambda_{2}\right)$ can be calculated within a two-fold summation

$$
S\left(\lambda_{1}, \lambda_{2}\right)=\sum_{j=1}^{\infty} \sum_{k=1}^{\infty} \frac{3}{(2 j+1)(2 j+3)} \frac{3}{(2 k+1)(2 k+3)} \frac{(2 j+2 k) !}{(2 j) !(2 k) !} \lambda_{1}^{2 j} \lambda_{2}^{2 k}
$$

\section{ANALYTICAL SHELL ENERGY}

The shape-dependent shell corrections have been determined within the Droplet Model formulae [30] with slightly different values of the parameters. The shell energy is :

$$
E_{\text {shell }}=E_{\text {shell }}^{\text {sphere }}\left(1-3.1 \theta^{2}\right) e^{-\theta^{2}} .
$$

The shell corrections for a spherical nucleus are given by

$$
E_{\text {shell }}^{\text {sphere }}=5.8\left[(F(N)+F(Z)) /(0.5 A)^{2 / 3}-0.28 A^{1 / 3}\right] M e V,
$$

where, for $M_{i-1}<X<M_{i}, M_{i}$ being the magic numbers,

$$
F(X)=q_{i}\left(X-M_{i-1}\right)-0.6\left(X^{5 / 3}-M_{i-1}^{5 / 3}\right)
$$

and

$$
q_{i}=0.6\left(M_{i}^{5 / 3}-M_{i-1}^{5 / 3}\right) /\left(M_{i}-M_{i-1}\right) .
$$

The assumed highest proton magic number is 114 while, for the two highest neutron magic numbers, the values 126 and 184 have been retained.

$$
\theta^{2}=(\delta R)^{2} / a^{2}
$$

The distortion $\theta a$ is the root mean square of the deviation of the nuclear surface from the sphere, a quantity which incorporates indiscriminately all types of deformation. The range $a$ has been chosen to be $0.286 r_{0}$.

For the two-body shapes, the total shell energy is the sum of the shell corrections for each deformed fragment.

This algebraic method to calculate the shell effects is questionable but it gives, at least for ellipsoidal deformations, almost the same results as the Strutinsky's method. Therefore, this approach seems justified for the ellipsoidal fragments and around the ground state. For large deformed one-body shapes the shell effects obtained by the Strutinsky's method are generally higher. 


\section{PAIRING ENERGY}

The pairing energy has been calculated with the following expressions provided by the Thomas-Fermi model [31]. For odd $\mathrm{Z}$, odd $\mathrm{N}$ and $\mathrm{N}=\mathrm{Z}$ nuclei

$$
E_{\text {Pairing }}=4.8 / N^{1 / 3}+4.8 / Z^{1 / 3}-6.6 / A^{2 / 3}+30 / A \text {. }
$$

For odd $\mathrm{Z}$, odd $\mathrm{N}$ and $N \neq Z$ nuclei

$$
E_{\text {Pairing }}=4.8 / N^{1 / 3}+4.8 / Z^{1 / 3}-6.6 / A^{2 / 3} .
$$

For odd Z, even N nuclei

$$
E_{\text {Pairing }}=4.8 / Z^{1 / 3} \text {. }
$$

For even Z, odd N nuclei

$$
E_{\text {Pairing }}=4.8 / N^{1 / 3} \text {. }
$$

For even Z, even N nuclei

$$
E_{\text {Pairing }}=0 \text {. }
$$

It is assumed that the pairing energy of the compound nucleus is constant before the separation and it is the sum of the pairing energy of the two fragments after the separation.

\section{POTENTIAL BARRIERS}

The potential barriers determined in the fusionlike fission valley within the macroscopic part of the GLDM (i.e without pairing and shell effects) and with the two sphere approximation for the fragments are plotted in Fig. 2 for the symmetric fission of $\beta$-stable nuclei. The proximity energy introduces progressively an inflection in the potential energy curve. For $A<215$ there is one saddle point corresponding to two separated spherical fragments in unstable equilibrium due to the balance between the repulsive Coulomb forces and the attractive nuclear proximity forces. For $A>215$, there are two maxima. The inner maximum is close to the sphere while the outer one presents always the same configuration ; between them a second minimum occurs due to the proximity effects in the neck which strongly lower the deformation energy. Naturally, these macroscopic double-humped barriers for actinides are not sufficient to reproduce the experimental data and the shell and pairing energies as well as the deformations of the fragments must be introduced.

The dependence of the deformation energy on the shape sequence and on the introduction of the microscopic corrections is displayed in Fig. 3 for an asymmetric fission path of the ${ }^{240} \mathrm{Pu}$ nucleus. The shell effects generate the slightly deformed ground state and contribute to the formation of the first peak. The proximity energy flattens the potential energy and the shell effects lead to the formation of a deep second minimum, lodging the observed superdeformed isomeric states. The value of the theoretical rigid moment of inertia, $2 I / \hbar^{2}$, of the ground state is $133 \mathrm{MeV}^{-1}$ relatively close to the experimental value of $140 \mathrm{MeV}^{-1}$ [1]. The value of the experimental moment of inertia of the isomeric state is $299 \mathrm{MeV}^{-1}$ while the theoretical one is $221 \mathrm{MeV}^{-1}$. The bottom of the second well is almost flat which may explain the difference between the two values.

The transition between one-body and two-body shapes is less smooth when the ellipsoidal deformations of the fragments are taken into account. Indeed, it corresponds to the transition (at $r=11.6$ fm for ${ }^{240} \mathrm{Pu}$ ) from a one-body quasi-molecular shape with spherical ends, a very deep neck and vanishing shell effects to two touching ellipsoidal fragments. The barrier height is reduced by several MeV. Later on, the magic Sn nucleus remains almost spherical while the other fragment evolves from an oblate shape to a prolate shape but the proximity energy keeps close the two tips of the fragments. A plateau appears in the potential energy curve at larger distances around $10 \mathrm{MeV}$ below the ground state. On this plateau the prolate deformation of the second fragment increases and the Coulomb and proximity energies diminish, the two fragments remaining in contact. The end of the plateau corresponds to the end of the contact between the two fragments and to a rapid transition from prolate to oblate or spherical shapes for the non-magical fragment and to the vanishing of the proximity energy, the two fragments going away.

It must be pointed out that the macro-microscopic models [32] and the Hartree-Fock-Bogoliubov approaches [33] have both difficulties to connect smoothly the two sheets of the potential energy surfaces corresponding respectively 


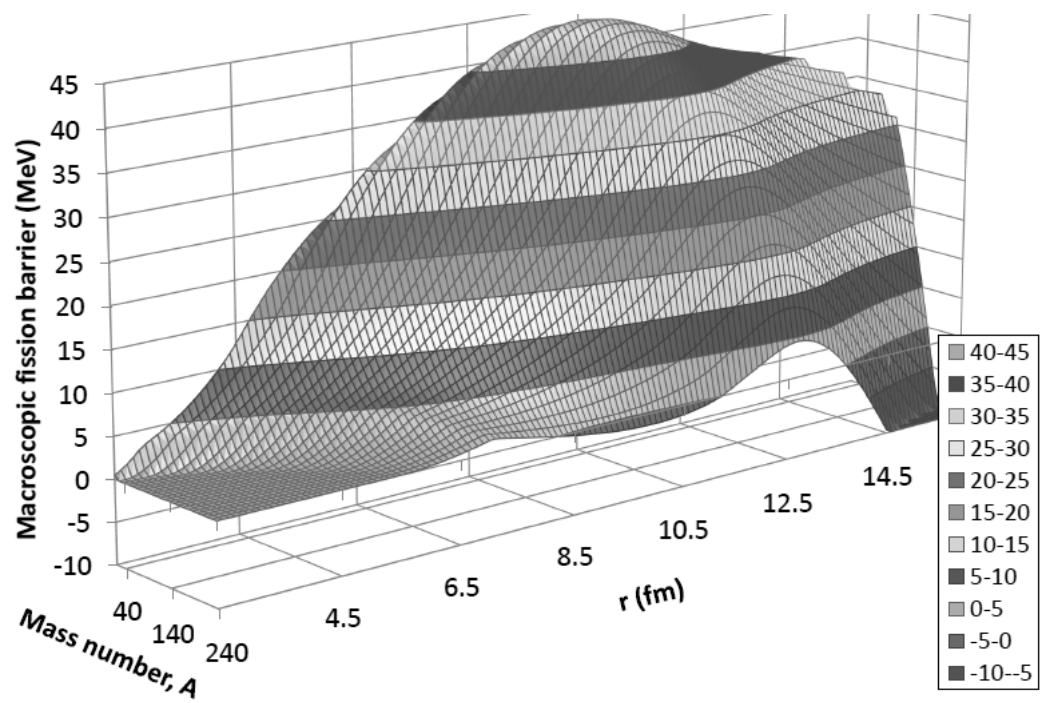

FIG. 2: Macroscopic barriers of the symmetric fission in the $\beta$ stability valley.

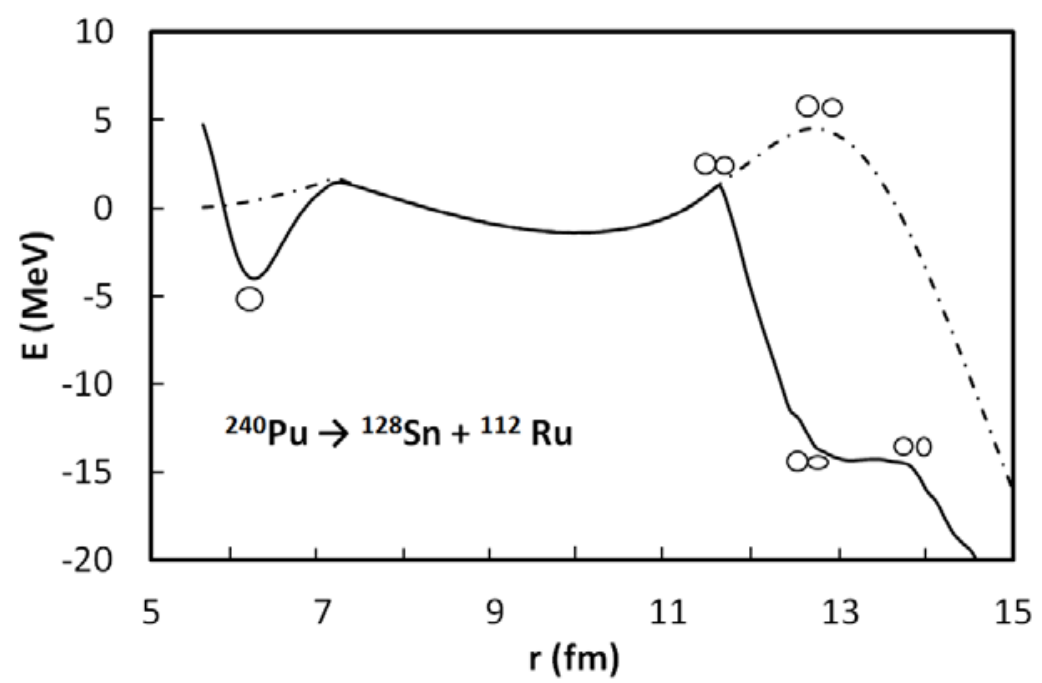

FIG. 3: Asymmetric fission barrier of a ${ }^{240} \mathrm{Pu}$ nucleus emitting a magic nucleus ${ }^{128} \mathrm{Sn}$. The dashed-dotted curve gives the macroscopic energy within the two-sphere approximation for the two-body shapes while the solid line includes the ellipsoidal deformations of the fragments and the shell and pairing energies. $\mathrm{r}$ is the distance between the mass centers.

to one-body and two-body shapes. Furthermore the models using elongated one-body shapes lead also generally to a second peak of the potential barrier which is too high.

When the proximity energy is not taken into account the scission point is often defined as the point where occurs the rupture of the matter bridge between the nascent fragments. In our approach this point is the second peak of the double-humped barrier but this is not the real scission point. The introduction of the proximity energy changes the definition of the scission point. Then, the scission point is the point where the nuclear attraction between the fragments vanishes. It corresponds in our approach to the end of the plateau where the proximity energy becomes negligible and the scission configuration is that of two well separated ellipsoids. The energy at the scission point is around $16 \mathrm{MeV}$ lower than the energy at the external saddle point. As in other studies, it exists a descent from the saddle-point till the scission point. The final fragment shell effects play a main role during this descent as underlined by Wilkins, Steinberg and Chasman long time ago [34]. Nevertheless, the fission fragment mass distribution is strongly connected with the relative half-lives of all the possible decays and, consequently, is mainly determined by the barriers all along the fission path. 
The selected one-body shape sequence depends only on two parameters and does not allow the investigation of all the possible nuclear shapes. Its interest lies in the efficiency to very rapidly reach the contact point between spherical or deformed fragments and to check the largest possible nuclear proximity effects. This quasi-molecular shape valley seems to be another possible candidate allowing the reproduction of the double-humped barriers of actinides.

In Fig. 4 the macro-microscopic double-humped barriers are displayed as a function of the mass of the heaviest fragment for the fission of the ${ }^{240} \mathrm{Pu}$ nucleus. For a given fragment mass, the charge which leads to the shortest decay half-life (see section VI) has been retained. The height of the inner peak is almost constant since it depends on the assumed shell effects after the ground state of the decaying nucleus. The height of the external peak generally increases with the asymmetry but the shell and pairing corrections induce strong distortions from this global behaviour. More precisely, the heights $E_{a}$ and $E_{b}$ of the two barriers are given in Fig. 5. The combination of the proton and neutron magic numbers respectively around 50 and 82 leads to lower the second barriers for masses of the heaviest fragment around 130 .

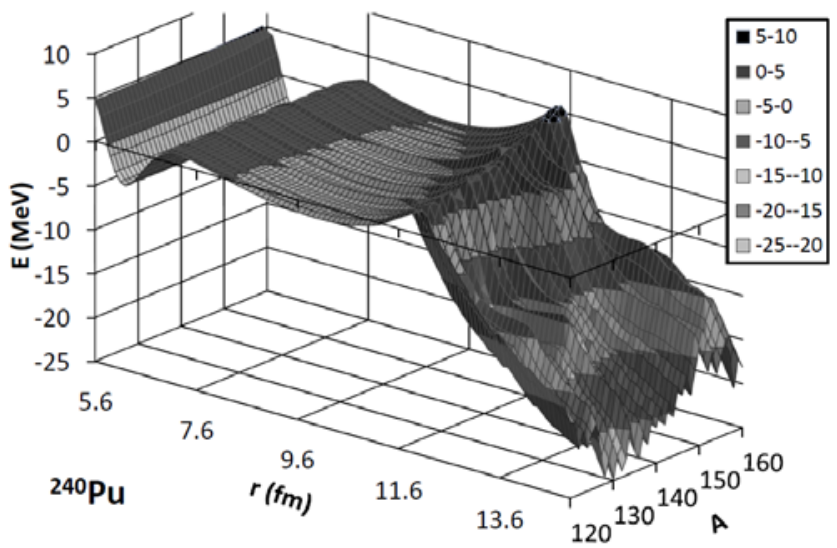

FIG. 4: Multiple-humped barriers for ${ }^{240} \mathrm{Pu}$ as a function of the heaviest fragment mass.

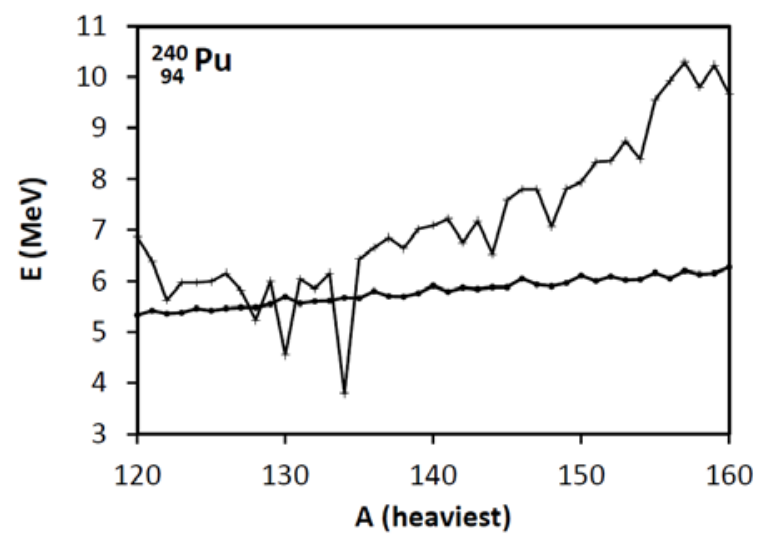

FIG. 5: Heights $E_{a}$ and $E_{b}$ of the inner (full circles) and outer (crosses) peaks of the fission barrier as a function of the mass of the heaviest fragment for ${ }^{240} \mathrm{Pu}$.

The 'experimental' fission barriers are usually extracted from induced fission excitation functions at excitation energy above the barrier. Hence, they correspond roughly to the lowest possible theoretical fission barriers. Within the GLDM, for each studied actinide isotope, the half-lives of all the possible decays have been calculated (see sect VI.). The decay giving the shortest half-life corresponds to the smallest action integral according to the method determining this half-life. This exit channel has been selected to compare the calculated and experimental energies of the maxima and minima of the fission barriers. The choice of this most probable fission path is difficult for some isotopes since there is a true degeneracy in energy between several paths. This problem comes from the fragment mass distribution which is very broad. The results are presented in Table 1. The experimental and theoretical heights 


\begin{tabular}{|c|c|c|c|c|c|c|c|c|c|}
\hline Reaction & $E_{a}(t)$ & $E_{a}(e)$ & $E_{2}(t)$ & $E_{2}(e)$ & $E_{b}(t)$ & $E_{b}(e)$ & $E_{3}(t h)$ & $E_{c}(t)$ & plateau energy \\
\hline${ }_{90}^{230} T h \rightarrow{ }_{50}^{132} S n+{ }_{40}^{98} Z r$ & 4.9 & - & 4.5 & - & 7.7 & - & 6.8 & 8.4 & 190 \\
\hline${ }_{90}^{231} \mathrm{Th} \rightarrow{ }_{50}^{132} \mathrm{Sn}+{ }_{40}^{99} \mathrm{Zr}$ & 5.0 & - & 4.5 & - & 7.4 & 6.5 & \begin{tabular}{|c|}
6.2 \\
e: 5.6 \\
\end{tabular} & \begin{tabular}{c|}
8.4 \\
e:6.3 \\
\end{tabular} & 191 \\
\hline${ }_{90}^{233} \mathrm{Th} \rightarrow{ }_{50}^{132} \mathrm{Sn}+{ }_{40}^{101} \mathrm{Zr}$ & 5.25 & - & 4.4 & - & 6.5 & 6.8 & \begin{tabular}{|c|}
5.1 \\
e: 5.2
\end{tabular} & \begin{tabular}{|c|}
7.65 \\
e: 6.8 \\
\end{tabular} & 189 \\
\hline${ }_{92}^{232} \mathrm{U} \rightarrow{ }_{52}^{134} \mathrm{Te}+{ }_{40}^{98} \mathrm{Zr}$ & 4.6 & 4.9 & 3.15 & - & 5.4 & 5.4 & 5.1 & 6.4 & 196 \\
\hline${ }_{92}^{234} \mathrm{U} \rightarrow{ }_{52}^{134} \mathrm{Te}+{ }_{40}^{100} \mathrm{Zr}$ & 5.0 & 5.6 & 3.2 & - & 4.8 & 5.5 & \begin{tabular}{|c|}
4.3 \\
e: 3.1
\end{tabular} & 6.3 & 195 \\
\hline${ }_{92}^{235} \mathrm{U} \rightarrow{ }_{50}^{131} \mathrm{Sn}+{ }_{42}^{104} \mathrm{Mo}$ & 5.4 & 5.7 & 4.2 & 2.5 & 6.1 & 5.8 & 5.35 & 7.15 & 199 \\
\hline${ }_{92}^{236} U \rightarrow{ }_{50}^{130} \mathrm{Sn}+{ }_{42}^{106} \mathrm{Mo}$ & 5.3 & 5.6 & 3.6 & $\begin{array}{c}2.3 \\
- \\
\end{array}$ & 5.0 & 5.5 & \begin{tabular}{|c|c|}
4.5 \\
e: 3.15 \\
\end{tabular} & 6.7 & 198 \\
\hline${ }_{92}^{237} U \rightarrow{ }_{50}^{132} \mathrm{Sn}+{ }_{42}^{105} \mathrm{Mo}$ & 5.8 & 6.1 & 4.6 & 2.5 & 6.2 & 5.9 & 4.9 & 7.1 & 199 \\
\hline${ }_{92}^{238} U \rightarrow{ }_{50}^{130} \mathrm{Sn}+{ }_{42}^{108} \mathrm{Mo}$ & 5.6 & 5.7 & 3.8 & 2.6 & 5.1 & 5.7 & 4.6 & 6.5 & 200 \\
\hline${ }_{92}^{239} U \rightarrow{ }_{50}^{132} \mathrm{Sn}+{ }_{42}^{107} \mathrm{Mo}$ & 5.9 & 5.8 & 4.4 & 1.9 & 5.6 & 6.0 & 4.0 & 6.2 & 197 \\
\hline${ }_{93}^{238} N p \rightarrow{ }_{50}^{132} S n+{ }_{43}^{106} T c$ & 6.0 & 6.0 & 4.9 & 2.3 & 6.6 & 6.0 & 5.6 & 7.1 & 205 \\
\hline${ }_{94}^{238} \mathrm{Pu} \rightarrow{ }_{52}^{132} \mathrm{Te}+{ }_{42}^{106} \mathrm{Mo}$ & 5.2 & 5.6 & 2.3 & 2.7 & 5.1 & 5.4 & & & 210 \\
\hline${ }_{94}^{239} \mathrm{Pu} \rightarrow{ }_{50}^{130} \mathrm{Sn}+{ }_{44}^{109} \mathrm{Ru}$ & 5.6 & 6.2 & 3.4 & 2.6 & 4.75 & 5.5 & 4.7 & 5.6 & 207 \\
\hline${ }_{94}^{240} \mathrm{Pu} \rightarrow{ }_{50}^{128} \mathrm{Sn}+{ }_{44}^{112} \mathrm{Ru}$ & 5.5 & 5.7 & 2.6 & 2.4 & 5.2 & 5.1 & & & 205 \\
\hline${ }_{94}^{241} \mathrm{Pu} \rightarrow{ }_{50}^{131} \mathrm{Sn}+{ }_{44}^{110} \mathrm{Ru}$ & 6.0 & 6.0 & 3.8 & 1.9 & 4.8 & 5.5 & 4.2 & 5.1 & 207 \\
\hline${ }_{94}^{243} \mathrm{Pu} \rightarrow{ }_{50}^{132} \mathrm{Sn}+{ }_{44}^{111} \mathrm{Ru}$ & 6.2 & 5.9 & 4.0 & 1.7 & 5.0 & 5.4 & 3.7 & 4.5 & 208 \\
\hline $\mathrm{g}_{95}^{242} \mathrm{Am} \rightarrow{ }_{50}^{131} \mathrm{Sn}+{ }_{45}^{111} \mathrm{Rh}$ & 6.1 & 6.5 & 3.85 & 2.9 & 5.2 & 5.4 & 4.8 & 5.25 & 214 \\
\hline${ }_{95}^{243} \mathrm{Am} \rightarrow{ }_{51}^{133} \mathrm{Sb}+{ }_{44}^{110} \mathrm{Ru}$ & 6.0 & 5.9 & 3.2 & 2.3 & 4.1 & 5.4 & 3.3 & 4.0 & 214 \\
\hline${ }_{95}^{244} \mathrm{Am} \rightarrow{ }_{50}^{132} \mathrm{Sn}+{ }_{45}^{112} \mathrm{Rh}$ & 6.3 & 6.3 & 4.0 & 2.8 & 5.1 & 5.4 & 3.9 & 4.3 & 218 \\
\hline $2_{96}^{243} \mathrm{Cm} \rightarrow{ }_{48}^{122} \mathrm{Cd}+{ }_{48}^{121} \mathrm{Cd}$ & 5.35 & 6.4 & 1.3 & 1.9 & 2.9 & 4.2 & & & 213 \\
\hline${ }_{96}^{245} \mathrm{Cm} \rightarrow{ }_{50}^{130} \mathrm{Sn}+{ }_{46}^{115} \mathrm{Pd}$ & 5.8 & 6.2 & 2.4 & 2.1 & 3.5 & 4.8 & & & 217 \\
\hline $2_{96}^{248} \mathrm{Cm} \rightarrow{ }_{50}^{130} \mathrm{Sn}+{ }_{46}^{118} \mathrm{Pd}$ & 5.8 & 5.7 & 2.0 & - & 2.7 & 4.6 & & & 216 \\
\hline${ }_{97}^{250} \mathrm{Bk} \rightarrow{ }_{50}^{130} \mathrm{Sn}+{ }_{47}^{120} \mathrm{Ag}$ & 5.7 & 6.1 & 1.3 & - & 2.0 & 4.1 & & & 222 \\
\hline${ }_{98}^{250} \mathrm{Cf} \rightarrow{ }_{49}^{125} \mathrm{In}+{ }_{49}^{125}$ In & 5.5 & 5.6 & 0.1 & - & 1.1 & - & & & 231 \\
\hline${ }_{99}^{256} \mathrm{Es} \rightarrow{ }_{50}^{128} \mathrm{Sn}+{ }_{49}^{128}$ In & 5.2 & 4.8 & -0.6 & - & -0.2 & - & & & 232 \\
\hline${ }_{100}^{255} \mathrm{Fm} \rightarrow{ }_{51}^{127} \mathrm{Sb}+{ }_{49}^{128} \mathrm{In}$ & 5.4 & 5.7 & -0.4 & - & 0.3 & - & & & 237 \\
\hline
\end{tabular}

TABLE I: Comparison between theoretical (t) and experimental (e) $[1,2,4,6]$ barrier characteristics for actinide nuclei. $E_{a}$, $E_{b}$ and $E_{c}$ are the first, second and third peak heights while $E_{2}$ and $E_{3}$ are the energies of the second and third potential minima, relatively to the ground state energy (in $\mathrm{MeV}$ ). The last column gives the energy of the plateau below the external barrier.

$E_{a}$ and $E_{b}$ of the two peaks roughly agree. The predicted value of the second minimum energy is often slightly too high. For the $\mathrm{U}, \mathrm{Pu}, \mathrm{Am}$ and $\mathrm{Cm}$ isotopes, an asymmetric path is favored. One fragment is close to the doubly magic ${ }_{50}^{132} \mathrm{Sn}$ nucleus, and, consequently, keeps an almost spherical shape. This effect does not appear for Cf, Es and Fm since for nuclei with $Z \sim 100$ the symmetric fission gives fragments with a charge close to the magic number 50. For the heaviest nuclei the external barrier disappears since the attractive proximity forces cannot compensate for the repulsive Coulomb forces any more.

The energy of the two deformed fragments at the end of the plateau below the multiple-humped barrier is given in the last column of Table 1. It follows the trend of the experimental distribution of the total kinetic of the fragments but it overestimates the data of about $20-30 \mathrm{MeV}$. That might be explained partially by the energy stored by collective excitation modes such as vibration, rotation, charge polarisation,...

A third shallow minimum and a third peak appear in certain asymmetric decay paths when the heaviest fragment is a magic nucleus which preserves its almost spherical shape. The non magic fragment was born in an oblate shape $(s \sim 1.4)$, due to the small distance between the mass centres at the transition between one-body and two-body shapes. When this distance increases, the ratio s decreases, because the proximity energy tends to keep close the two tips of the fragments. The lightest fragment remaining in contact with the other spherical fragment approaches the spherical shape and its shell energy increases to reach a maximum, which is at the origin of the third peak and corresponds to two touching different spheres. Before reaching this third peak a third shallow minimum appears. Its shape is hyperdeformed and asymmetric, in agreement with the experimental data [4]. Later on, the proximity forces maintain the two fragments in contact and the shape of the smallest one evolves to prolate shapes $(s<1)$, the corresponding shell corrections decreasing. In the symmetric mass exit path, the proximity and Coulomb energies 
counterbalance the smallest shell effects and induce an asymmetric shape, the two fragments remain in contact but one fragment is oblate while the other one is prolate. With increasing distance between the mass centres the two nuclei become prolate. There is no third barrier in the symmetric deformation paths. The still sparse and controversial data for the third barrier are roughly reproduced.

Clearly, the magicity of the proton and neutron numbers of the fragments plays a main role to discriminate between all the possible exit channels. Different hypotheses have been checked for the calculations of the pairing energy : pairing depending on the shape, pairing independent of the shapes but different for one and two-body shapes and no pairing term. There are fluctuations but the global agreement is about the same.

\section{HALF-LIVES}

\begin{tabular}{|c|c|c|}
\hline Reaction & $T_{1 / 2, \exp (s)}$ & $T_{1 / 2, \text { theo }(s)}$ \\
\hline${ }_{92}^{232} \mathrm{U} \rightarrow{ }_{52}^{134} \mathrm{Te}+{ }_{40}^{98} \mathrm{Zr}$ & $2.5 \times 10^{21}$ & $4.8 \times 10^{18}$ \\
\hline${ }_{92}^{234} \mathrm{U} \rightarrow{ }_{52}^{134} \mathrm{Te}+{ }_{40}^{100} \mathrm{Zr}$ & $4.7 \times 10^{23}$ & $4.6 \times 10^{19}$ \\
\hline${ }_{92}^{235} \mathrm{U} \rightarrow{ }_{50}^{131} \mathrm{Sn}+{ }_{42}^{104} \mathrm{Mo}$ & $3.1 \times 10^{26}$ & $1.1 \times 10^{24}$ \\
\hline${ }_{92}^{236} \mathrm{U} \rightarrow{ }_{50}^{130} \mathrm{Sn}+{ }_{42}^{106} \mathrm{Mo}$ & $7.8 \times 10^{23}$ & $1.7 \times 10^{22}$ \\
\hline${ }_{92}^{238} \mathrm{U} \rightarrow{ }_{50}^{130} \mathrm{Sn}+{ }_{42}^{108} \mathrm{Mo}$ & $2.6 \times 10^{23}$ & $5.2 \times 10^{23}$ \\
\hline${ }_{94}^{238} \mathrm{Pu} \rightarrow{ }_{52}^{132} \mathrm{Te}+{ }_{42}^{106} \mathrm{Mo}$ & $1.5 \times 10^{18}$ & $2.1 \times 10^{18}$ \\
\hline${ }_{94}^{239} \mathrm{Pu} \rightarrow{ }_{50}^{130} \mathrm{Sn}+{ }_{44}^{109} \mathrm{Ru}$ & $2.5 \times 10^{23}$ & $10^{22}$ \\
\hline${ }_{94}^{240} \mathrm{Pu} \rightarrow{ }_{50}^{128} \mathrm{Sn}+{ }_{44}^{112} \mathrm{Ru}$ & $3.7 \times 10^{18}$ & $2.3 \times 10^{20}$ \\
\hline${ }_{95}^{243} \mathrm{Am} \rightarrow{ }_{51}^{133} \mathrm{Sb}+{ }_{44}^{110} \mathrm{Ru}$ & $6.3 \times 10^{21}$ & $3.6 \times 10^{22}$ \\
\hline${ }_{96}^{243} \mathrm{Cm} \rightarrow{ }_{48}^{122} \mathrm{Cd}+{ }_{48}^{121} \mathrm{Cd}$ & $1.7 \times 10^{19}$ & $2.3 \times 10^{16}$ \\
\hline${ }_{96}^{245} \mathrm{Cm} \rightarrow{ }_{50}^{130} \mathrm{Sn}+{ }_{46}^{115} \mathrm{Pd}$ & $4.4 \times 10^{19}$ & $2.0 \times 10^{20}$ \\
\hline${ }_{96}^{248} \mathrm{Cm} \rightarrow{ }_{50}^{130} \mathrm{Sn}+{ }_{46}^{118} \mathrm{Pd}$ & $1.3 \times 10^{14}$ & $1.9 \times 10^{18}$ \\
\hline${ }_{98}^{250} C f \rightarrow{ }_{49}^{125}$ In $+{ }_{49}^{125}$ In & $5.2 \times 10^{11}$ & $4.2 \times 10^{11}$ \\
\hline${ }_{99}^{255} \mathrm{Es} \rightarrow{ }_{50}^{128} \mathrm{Sn}+{ }_{49}^{127} \mathrm{In}$ & $8.4 \times 10^{10}$ & $5.5 \times 10^{6}$ \\
\hline${ }_{100}^{256} \mathrm{Fm} \rightarrow{ }_{50}^{128} \mathrm{Sn}+{ }_{50}^{128} \mathrm{Sn}$ & $1.0 \times 10^{4}$ & $1.1 \times 10^{4}$ \\
\hline${ }_{102}^{256} \mathrm{No} \rightarrow{ }_{51}^{128} \mathrm{Sb}+{ }_{51}^{128} \mathrm{Sb}$ & 110 & $1.9 \times 10^{0}$ \\
\hline
\end{tabular}

TABLE II: Comparison between experimental [2] and theoretical spontaneous fission half-lives of actinide nuclei.

Within this asymmetric fission model the decay constant is simply given by $\lambda=\nu_{0} P$. The assault frequency $\nu_{0}$ has been taken as $\nu_{0}=10^{20} \mathrm{~s}^{-1}$. The barrier penetrability $\mathrm{P}$ is calculated within the action integral

$$
P=\exp \left[-\frac{2}{\hbar} \int_{r_{\text {in }}}^{r_{\text {out }}} \sqrt{2 B(r)\left(E(r)-E_{g . s}\right)} d r\right] .
$$

The inertia $\mathrm{B}(\mathrm{r})$ is related to the reduced mass by

$$
B(r)=\mu\left[1+24 \exp \left(-3.25\left(r-R_{s p h}\right) / R_{0}\right)\right]
$$

where $R_{s p h}$ is the distance between the mass centers of the future fragments in the initial sphere, $R_{s p h} / R_{0}=0.75$ in the symmetric case.

For shapes near the ground state the inertia is largely above the irrotational flow value since a large amount of internal reorganization occurs at level crossings. For highly deformed shapes the reduced mass is reached asymptotically.

The partial half-life is finally obtained by $T_{1 / 2}=\frac{\ln 2}{\lambda}$.

The experimental spontaneous fission half-lives and theoretical predictions for the assumed most probable exit channels are compared in Table 2. There is a rough agreement with most of the experimental data on 26 orders of magnitude. Let us recall that the same GLDM and method to determine the half-lives have allowed an accurate reproduction of the alpha and cluster radioactivity half-lives [18, 19].

\section{SUMMARY AND CONCLUSION}

Exit channels of actinides via the quasi-molecular shape valley have been investigated within a generalized liquid drop model including the nuclear proximity energy and microscopic corrections. Double-humped potential barriers 
and large deformed minima lodging possibly isomeric states appear. The external saddle-point corresponds to the transition from one-body shapes to two touching ellipsoids. The scission point, where the effects of the nuclear attractive forces between the fragments vanish, lies at the end of an energy plateau below the saddle-point. It corresponds to two well separated fragments. The energy on this plateau gives the fragment kinetic energy plus an excitation energy. The barrier heights roughly agree with the experimental results on the double-humped fission barriers. The shell and pairing effects play a main role to decide the most probable decay path. The predicted half-lives follow most of the experimental data.

A shallow third minimum and a third peak appear in specific asymmetric exit channels where one fragment is close to a double magic quasi-spherical nucleus, while the other one evolves from oblate to prolate shapes.

[1] S. Björnholm and J.E. Lynn, Rev. Mod. Phys. 52, 725 (1980).

[2] C. Wagemans, The nuclear fission process (CRC Press, Boca Raton, 1991).

[3] A. Krasznahorkay et al., Phys. Rev. Lett. 80, 2073 (1998).

[4] A. Krasznahorkay et al., Phys. Lett. B 461, 15 (1999).

[5] M. Hunyadi et al., Phys. Lett. B 505, 27 (2001).

[6] J. Blons, C. Mazur, D. Paya, M. Ribrag and H. Weigmann, Nucl. Phys. A 414, 1 (1984).

[7] A. Marinov, S. Gelberg, D. Kolb, R. Brandt and A. Pape, Int. J. Mod. Phys. E 12, 1 (2003).

[8] P. Armbruster, Eur. Phys. J. A 37, 159 (2008).

[9] V. Zagrebaev and W. Greiner, Phys. Rev. C 78, 034610 (2008).

[10] V.M. Strutinsky, Nucl. Phys. A 95, 420 (1967).

[11] W.D. Myers and W.J. Swiatecki, Ark. Fys. 36, 343 (1967).

[12] M.G. Mustafa, U. Mosel and H.W. Schmitt, Phys. Rev. C 7, 1519 (1973).

[13] J.F. Berger, M. Girod and D. Gogny, J. Physique Lett. 42, 509 (1981).

[14] V. Blum, J.A. Maruhn, P.G. Reinhard and W. Greiner, Phys. Lett. B 323, 262 (1994).

[15] G. Royer and B. Remaud, J. Phys. G : Nucl. Phys. 10, 1541 (1984).

[16] G. Royer anf F. Haddad, Phys. Rev. C 51, 2813 (1995).

[17] G. Royer and K. Zbiri, Nucl. Phys. A 697, 630 (2002).

[18] G. Royer, J. Phys. G 26, 1149 (2000).

[19] G. Royer and R. Moustabchir, Nucl. Phys. A 683, 182 (2001).

[20] G. Royer and B. Remaud, Nucl. Phys. A 444, 477 (1985).

[21] G. Royer, C. Bonilla and R. A. Gherghescu, Phys. Rev. C 67, 034315 (2003).

[22] G. Royer and J. Gaudillot, Phys. Rev. C 84, 044602 (2011).

[23] J. Mignen, G. Royer and F. Sebille, Nucl. Phys. A 489, 461 (1988).

[24] H. Feldmeier, $12^{t h}$ Summer School on nuclear physics, Mikolajki, Poland, 1979.

[25] S. Cohen and W.J. Swiatecki, Ann. of Phys. 22, 406 (1963).

[26] J. Mignen and G. Royer, J. Phys. G : Nucl. Part. Phys. 16, L227 (1990).

[27] J. Blocki, J. Randrup, W.J. Swiatecki and C.F. Tsang, Ann. of Phys. 105, 427 (1977).

[28] G. Royer, M. Guilbaud and A. Onillon, Nucl. Phys. A 847, 24 (2010).

[29] G. Royer and C. Piller, J. Phys. G : Nucl. Phys. 18, 1805 (1992).

[30] W.D. Myers, Droplet model of atomic nuclei (Plenum, New-York, 1977).

[31] W.D. Myers and W.J. Swiatecki, Nucl. Phys. A 601, 141 (1996).

[32] P. Möller, A.J. Sierk and A. Iwamoto, Phys. Rev. Lett. 92, 072501 (2004).

[33] J.F. Berger, M. Girod and D. Gogny, Nucl. Phys. A 502, 85c (1989).

[34] B.D. Wilkins, E.P. Steinberg and R.R. Chasman, Phys. Rev. C 14, 1832 (1976). 